\title{
Megacolon tóxico secundario a enfermedad de Hirschsprung. Reporte de un caso
}

\author{
Toxic megacolon secondary to Hirschsprung's disease. A case report
}

\author{
Jorge Arturo Soto Pérez, ${ }^{*}$ Roberto Ledesma Briseño, ${ }^{* *}$ Roberto Pablo Martínez Rubio, ${ }^{* * *}$ \\ Ricardo Reynoso González,**** Jesús San Román Sánchez*****
}

\begin{abstract}
Palabras clave:
Hirschsprung,

megacolon tóxico.
\end{abstract}

Key words:

Hirschsprung, toxic megacolon.

\section{* Médico Especialista en Cirugía General y Coloproctología. Adscrito al Servicio de Coloproctología en el Hospital Regional "Lic. Adolfo López Mateos", ISSSTE. \\ ** Médico Especialista en Cirugía General. Jefe del Servicio de Cirugía General en el Hospital General de Zona \#98, IMSS. \\ *** Médico Especialista en Cirugía General y residente de $4^{\circ}$ año de Cirugía Cardiotorácica en el Centro Médico "20 de Noviembre", ISSSTE. ***** Médico Especialista en Cirugía General. Adscrito al Servicio de Cirugía General en el Hospital General de Zona \#68, IMSS. \\ ***** Médico \\ Especialista en Cirugía General. Adscrito al \\ Servicio de Cirugía General en el Hospital Regional de Alta Especialidad de Zumpango, ISEM.}

\section{RESUMEN}

La enfermedad de Hirschsprung, descrita en 1888, es definida como la presencia de menos de $20 \%$ de células ganglionares normales por milímetro de intestino. Algunos pacientes pueden cursar con megacolon como complicación. Presentamos el caso de un masculino de 46 años con estreñimiento crónico, con ausencia de evacuaciones durante un mes, acompañado de vómito, distensión abdominal y datos de toxicidad. Presentaba abdomen agudo y se realizó laparotomía exploradora, con la que se encontró dilatación del sigmoides que abarcaba la totalidad de la cavidad abdominal; se realizó hemicolectomía izquierda, con colostomía a nivel del transverso. Evolucionó de una manera tórpida y se reintervino al sexto día para realizar colectomía más ileostomía por presentar nuevamente distensión importante del resto del colon. Se envió la pieza quirúrgica a estudio histopatológico y reportaron agangliosis; se diagnosticó enfermedad de Hirschsprung. El caso que se presenta es sumamente raro debido a que la enfermedad de Hirschsprung es congénita, se diagnostica frecuentemente en la infancia; cuando no es de esta manera, se presenta en el adulto con los síntomas mencionados; sin embargo, el paciente cursó con megacolon tóxico, una situación inusual.

\section{ABSTRACT}

Hirschsprung's disease, described in 1888, is defined as the presence of less than $20 \%$ of normal ganglion cells per milliliter of intestine. Some patients may present with megacolon as a complication. We present the case of a 46-year-old male with chronic constipation, with no evacuations for one month, accompanied by vomiting, abdominal distension and toxicity data. He presented with acute abdomen; we performed an exploratory laparotomy, in which we found dilatation of the sigmoid that covered the whole abdominal cavity; left hemicolectomy was performed, with colostomy at the transverse level. It evolved in a torpid way and was intervened on the sixth day to perform a colectomy plus ileostomy for presenting again significant distention of the rest of the colon. The surgical specimen was sent to histopathological study and reported agangliosis, diagnosing Hirschsprung's disease. The case presented is extremely rare because Hirschsprung's disease is congenital, it is often diagnosed in childhood; when it is not, it occurs in the adult with the symptoms mentioned; however, the patient coursed with toxic megacolon, an unusual situation.

\section{INTRODUCCIÓN}

L a enfermedad de Hirschsprung (EH) fue descrita por primera vez en 1888 , en dos lactantes, por el pediatra de Copenhague Harald Hirschsprung. Se trataba de un caso con estreñimiento grave acompañado de una dilatación e hipertrofia del colon. No se encontró una obstrucción mecánica que justificara el estreñimiento y la zona dilatada; se consideró como el asiento primario de la enfermedad..$^{1-4}$

La EH tiene una incidencia de uno en 500 neonatos en la literatura occidental y predo- mina en hombres sobre mujeres $4: 1 .^{1-4}$ Esta relación disminuye 2:1 cuando el segmento afectado es más largo. ${ }^{2-4}$

La EH se define como la presencia menor o igual al $20 \%$ de células ganglionares normales por milímetro de intestino..$^{2,3}$ La enfermedad de Hirschsprung en el adulto es relativamente rara porque se diagnostica en la infancia; en algunos debuta con megacolon. ${ }^{4,5}$

La EH puede ser clasificada en: 1) segmento corto, cuando no compromete más allá de la unión rectosigmoidea; 2) ultracorto, si sólo afecta el esfínter interno y/o algunos milímetros proximales a él; y 3) segmento largo, cuando 
el segmento agangliónico afecta más allá de la unión rectosigmoidea. ${ }^{1,2,4,5}$

La enfermedad de Hirschsprung del adulto es una patología poco frecuente y subdiagnosticada que se manifiesta con periodos prolongados de constipación refractaria, con una incidencia cercana al $0.002 \%$ de la población adulta. ${ }^{3-5}$ La patogenia se explica por una falla en la migración craneocaudal de las células ganglionares provenientes de la cresta. Histológicamente, este padecimiento muestra hiperplasia de los troncos nerviosos que contienen acetilcolinesterasa y ausencia total de los elementos neuronales que producen sintetasa de óxido nítrico. ${ }^{6-8}$ El diagnóstico puede ser sospechado con colon por enema, manometría anorrectal, y confirmarse con el estándar de oro, biopsia. La colonoscopia puede utilizarse en caso de megacolon, con el fin de resolver la urgencia y realizar un tratamiento "electivo". ${ }^{9-11}$ Los procedimientos quirúrgicos más aceptados en la literatura médica son el de Swenson, Duhamel, Soave y cirugía de Lynn. ${ }^{12}$

El objetivo del siguiente trabajo es presentar un caso, su abordaje diagnóstico y terapéutico, así como la revisión de la literatura.

\section{REPORTE DEL CASO}

Masculino de 46 años con antecedente de estreñimiento crónico desde los 14 años, en manejo empírico con laxantes dos veces por día, tabaquismo de 20 años de evolución y etilismo. Inició su padecimiento un mes previo con ausencia de evacuaciones, agregándose progresivamente dolor abdominal crónico que se agudizó y generalizó dos días previos a su ingreso, acompañado de náusea y vómito gastroalimentario en ocho ocasiones, anorexia, distensión abdominal e imposibilidad para canalizar gases. A su ingreso a urgencias, sus signos vitales fueron: temperatura, 36.2; frecuencia cardiaca, 120 latidos por minuto; frecuencia respiratoria, 24 por minuto; tensión arterial, 90/60 $\mathrm{mmHg}$. A la exploración física, se le observó pálido, mal hidratado, con abdomen globoso, timpanismo generalizado con pérdida de la matidez hepática, dolor a la palpación media y profunda en todos los cuadrantes, resistencia muscular involuntaria y descompresión positiva (Figura 1). A su ingreso, los estudios de laboratorio fueron los descritos en el cuadro I.

En la radiografía simple de abdomen, se apreciaba una imagen correspondiente al colon sigmoides, el cual se observó con abundante materia fecal en su interior y abarcaba la totalidad de la cavidad abdominal, con borramiento de la silueta hepática e imagen en vidrio despulido (Figura 2). En la radiografía de tórax, se apreció el hemidiafragma izquierdo desplazado cefálicamente por un asa intestinal (Figura 3). Se realizó laparotomía exploradora, donde se encontró sigmoides a tensión con pared intestinal engrosada, abarcando la totalidad de la cavidad abdominal (Figura 4), manteniendo el resto del intestino desplazado de manera posterior, el hígado y bazo hacia la región superior del abdomen y el diafragma izquierdo elevado. Se realizó colotomía descompresiva y se drenó abundante materia fecal y gas (Figura 5);

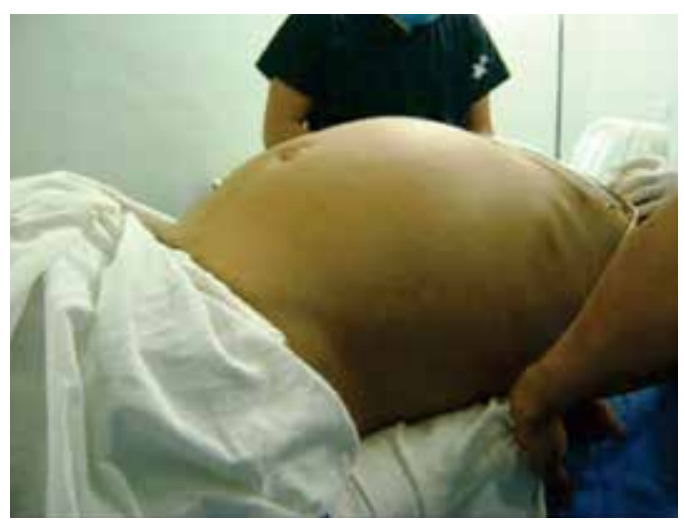

Figura 1. Abdomen distendido y a tensión.

\section{Cuadro I. Resultados de los estudios al ingreso.}

Leucocitos 12,900/ml Urea $98.4 \mathrm{mg} / \mathrm{dl}$ Neutrófilos 73.4\% Nitrógeno ureico 46 $\mathrm{mg} / \mathrm{dl}$

Eritrocitos $4.83 \times 10^{3} \mu \mathrm{l}$ Creatinina $2.2 \mathrm{mg} / \mathrm{dl}$ Hemoglobina $7.7 \mathrm{~g} / \mathrm{dl} \quad$ Bilirrubina total 1.20 $\mathrm{mg} / \mathrm{dl}$

Hematocrito 29.2\% El resto, sin alteraciones 


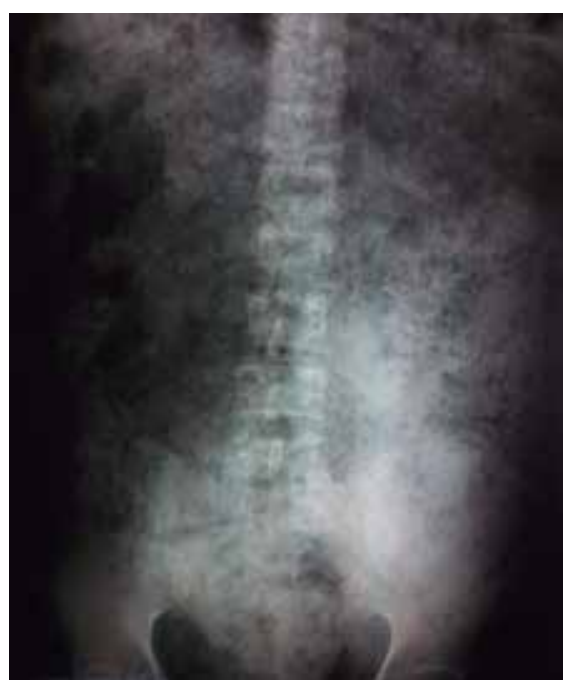

Figura 2. Radiografía simple de abdomen. Materia fecal y gas de sigmoides ocupando la totalidad de la cavidad abdominal.

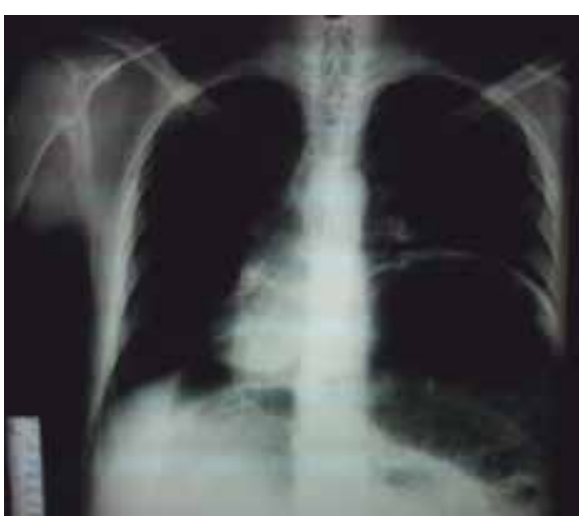

Figura 3. Telerradiografía de tórax. Sigmoides distendido y elevando el hemidiafragma izquierdo.

se procedió a la hemicolectomía izquierda y se dejó colostomía del transverso. El paciente fue ingresado a terapia intensiva por choque hipovolémico grado III y sepsis abdominal; evolucionó de manera tórpida y sin gasto a través de la colostomía. Requirió de una nueva intervención al sexto día, y presentó nueva dilatación del colon transverso y ascendente, por lo que se realizó colectomía total con ileostomía. Fue manejado de forma conservadora; egresó a los 10 días de la terapia intensiva y a los 18 días de piso de cirugía con la ileostomía funcional.

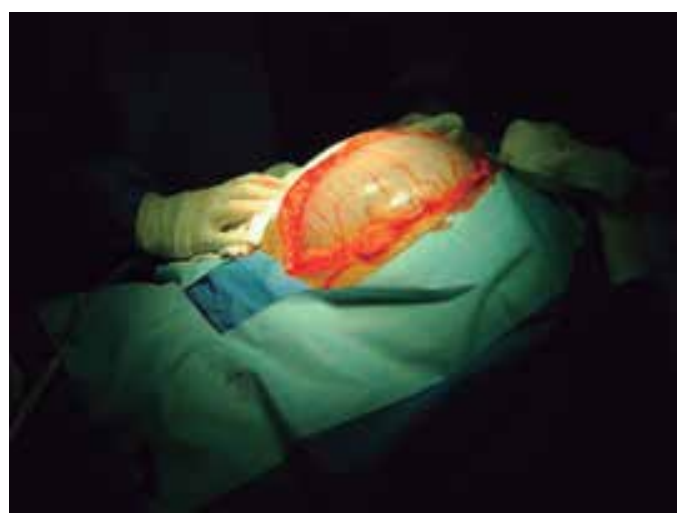

Figura 4. Laparotomía exploradora donde se observa el sigmoides ocupando toda la cavidad abdominal.

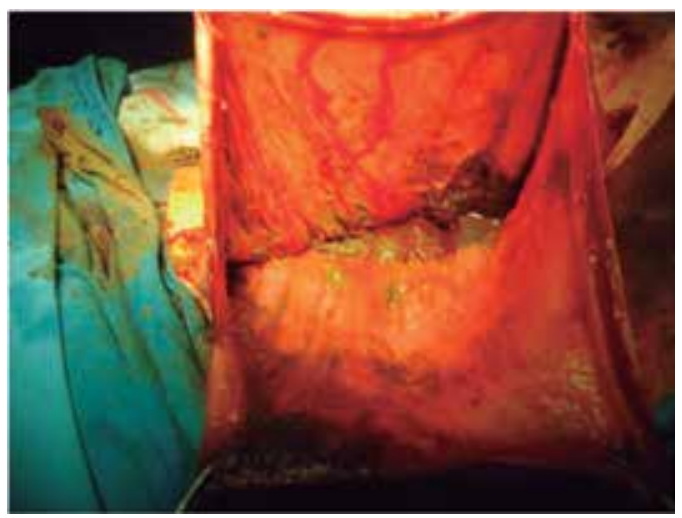

Figura 5. Colotomía del sigmoides ya drenado.

\section{DISCUSIÓN}

La mayoría de los pacientes son diagnosticados con EH durante su adolescencia o el inicio de la vida adulta. No está claro si la forma adulta de la enfermedad se debe a una falla en el diagnóstico durante la infancia o si realmente es una presentación tardía de la enfermedad. 3,4

De cualquier modo, las formas medianas de la enfermedad pueden ser no diagnosticadas y progresar hasta la vida adulta, probablemente porque la región colónica proximal al segmento obstruido asume un papel compensatorio. ${ }^{4,5} \mathrm{El}$ paciente que se presenta estuvo diagnosticado desde su infancia con estreñimiento crónico y fue tratado con diversos laxantes, presentaba evacuaciones cada semana y obtenía un resultado parcialmente satisfactorio. En el caso expuesto, es probable que el segmento colónico proximal dilatado se 
haya descompensado debido a una obstrucción fisiológica distal y, consecutivamente, presentado un rápido empeoramiento de la constipación de una forma aguda, hecho que llevó al colon del paciente a presentar una dilatación importante y, luego, datos de toxicidad; en consecuencia, se dieron los planes terapéuticos ya descritos.

Es poco mencionada la asociación de megacolon tóxico con enfermedad de Hirschsprung en adultos; sin embargo, en niños es más frecuente que exista, siendo la EH la causa más frecuente de megacolon tóxico en infantes y niños. ${ }^{6,7}$ En la literatura se encuentran pocos casos reportados de megacolon tóxico secundario a $\mathrm{EH}$ en el adulto.

El paciente comentado cuenta con los criterios de megacolon tóxico expuestos por Jalan K. y colaboradores: diámetro $>6 \mathrm{~cm}$ en la radiografía de abdomen, deshidratación, trastornos hidroelectrolíticos, leucocitosis, distensión abdominal, anemia e hipotensión. En los casos reportados en la literatura médica, es común que se intervengan quirúrgicamente los pacientes por megacolon tóxico secundario a $\mathrm{EH}$, debido a que no se tiene clara la presencia de la enfermedad y ésta se confirma con el estudio de la pieza quirúrgica. ${ }^{6,11,13}$

\section{CONCLUSIONES}

Reportamos el caso de un paciente adulto con megacolon tóxico secundario a $\mathrm{EH}$, una complicación sumamente rara de dicho padecimiento. Es importante, para realizar el diagnóstico, la sospecha clínica basada en los antecedentes del paciente y los hallazgos de la cirugía. Sin embargo, para dar un diagnóstico certero, es necesario el estudio de la pieza quirúrgica y confirmar la ausencia de los plexos entéricos o aumento de los troncos nerviosos con acetilcolinesterasa. En pacientes que se presentan con megacolon tóxico secundario a EH, la posibilidad más loable es intervenir quirúrgicamente de urgencia para resolver la oclusión y realizar una derivación digestiva, para luego estudiar el caso, siempre teniendo la sospecha del padecimiento y sus formas de presentación.

\section{REFERENCIAS}

1. Peña A, Levitt MA. Pediatric surgical problems. In: Corman ML, editor. Corman's colon and rectal surgery. 6th ed. Philadelphia: Lippincott Williams \& Wilkins; 2013. p. 556-569.

2. Teitelbaum DH, Ehrlich PF. Pediatric colorectal disorders. In: Steele SR, Hull TL, Read TE, Saclarides TJ, Senagore AJ, Whitlow CB, editors. The ASCRS textbook of colon and rectal surgery. 3rd ed. Illinois: Springer; 2016. p. 1140-1142.

3. Qiu JF, Shi YJ, Hu L, Fang L, Wang HF, Zhang MC. Adult Hirschsprung's disease: report of four cases. Int J Clin Exp Pathol. 2013; 6: 1624-1630.

4. Bakari AA, Gali BM, Ibrahim AG, Nggada HA, Ali N, Dogo D, et al. Congenital aganglionic megacolon in Nigerian adults: two case reports and review of the literature. Niger J Clin Pract. 2011; 14: 249-252.

5. Vorobyov GI, Achkasov SI, Biryukov OM. Clinical features' diagnostics and treatment of Hirschsprung's disease in adults. Colorectal Dis. 2010; 12: 12421248.

6. Alagumuthu M, Jagdish S, Kadambari D. Hirschsprung's disease in adults presenting as sigmoid volvulus: a report of three cases. Trop Gastroenterol. 2011; 32: 341-343.

7. Bălănescu RN, Bălănescu L, Moga AA, Drăgan GC, Djendov FB. Segmental aganglionosis in Hirschsprung's disease in newborns - a case report. Rom J Morphol Embryol. 2015; 56: 533-536.

8. Agrawal RK, Kakkar N, Vasishta RK, Kumari V, Samujh R, Rao KL. Acetylcholinesterase histochemistry (AchE) -A helpful technique in the diagnosis and in aiding the operative procedures of Hirschsprung disease. Diagn Pathol. 2015; 10: 208.

9. Luzón-Toro B, Espino-Paisán L, Fernández RM, MartínSánchez M, Antiñolo G, Borrego S. Next-generationbased targeted sequencing as an efficient tool for the study of the genetic background in Hirschsprung patients. BMC Med Genet. 2015; 16: 89.

10. Tang YF, Chen JG, An HJ, Jin P, Yang L, Dai ZF, et al. High-resolution anorectal manometry in newborns: normative values and diagnostic utility in Hirschsprung disease. Neurogastroenterol Motil. 2014; 26: 15651572.

11. Swaminathan M, Oron AP, Chatterjee S, Piper H, CopeYokoyama S, Chakravarti A, et al. Intestinal neuronal dysplasia-like submucosal ganglion cell hyperplasia at the proximal margins of Hirschsprung disease resections. Pediatr Dev Pathol. 2015; 18: 466-476.

12. Zhang S, Li J, Wu Y, Hu Y, Duan C, Wang M, et al. Comparison of laparoscopic-assisted operations and laparotomy operations for the treatment of Hirschsprung disease: evidence from a meta-analysis. Medicine (Baltimore). 2015; 94: e1632.

13. Autenrieth DM, Baumgart DC. Toxic megacolon. Inflamm Bowel Dis. 2012; 18: 584-591.

Correspondencia:

Dr. Jorge Arturo Soto Pérez

Hospital Regional "Lic. Adolfo López Mateos". Av. Universidad Núm. 1321,

Col. Florida, Del. Álvaro Obregón, 01030, Ciudad de México, México. Teléfonos: 53-22-23-00, ext. 89371

Teléfono directo: 53-22-26-39

E-mail: dropafett@gmail.com 